\title{
Percepção dos acadêmicos sobre a vivência de fisioterapia na atenção primária a saúde
}

\author{
Maíra Machado da Silva*, Camila dos Santos Dias**, Danuza \\ Teixeira Corrêa*, Êmille dos Santos ${ }^{\star \star *}$, Maria Saleti Lock Vogt ${ }^{\star \star \star *}$, \\ Stéphanie Jesien**
}

RESUMO: Este trabalho objetiva relatar a percepção dos acadêmicos sobre a vivência na disciplina de Fisioterapia na Atenção Primária. Metodologia: o presente estudo desenvolveu-se na disciplina teóricoprática "Fisioterapia na Atenção Primária a Saúde", com carga horária de 30 horas. As práticas foram desenvolvidas na comunidade da Estratégia Saúde da Família - S. José, em Santa Maria/RS. Participaram 14 acadêmicos do curso de fisioterapia da Universidade Federal de Santa Maria. Os resultados são oriundos do parecer dos acadêmicos sobre a vivência. Procedeu-se uma leitura qualitativa dos dados, tendo como referencial de análise a sistematização proposta por Minayo'. Resultados: Os resultados estão apresentados em três blocos, de acordo com os itens do parecer: facilidades para aprendizagem, dificuldades e sugestões para as dificuldades, e auto-avaliação sobre a experiência. Conclusão: Observou-se a satisfação dos acadêmicos com a experiência, que permitiuIhes refletir sobre a importância da inclusão do fisioterapeuta na atenção primária a saúde.

Descritores: Atenção primária à saúde, Educação em saúde, Fisioterapia.

\section{Students' perception on the experience of physical therapy in primary health care}

ABSTRACT: This paper describes perception of academic students about experience in the discipline of Physical Therapy in Primary Care. Methods: this present study in the theoretical-practical discipline "Physical Therapy in Primary Care", with a workload of 30 hours. The practices were developed in the community of the Family Health Strategy- São José, Santa Maria, RS. Participated 14 Physical Therapy students of the University Federal of Santa Maria. The results come from the feedback of scholars about the experience. Procedure a qualitative data analysis, referencing the systematization analysis proposed by Minayo Results: The results were presented in three categories, according to the items of the feedback: facilities for learning, difficulties and suggestions for the difficulties, and self-evaluation of the experience. Conclusion: Observed Satisfaction of the students with the experience, which allowed them to reflect about the importance of including the physiotherapist in the primary healthcare.

Descriptors: Primary health care, Health education, Physiotherapy.

\footnotetext{
*Fisioterapeuta. Especializanda em Reabilitação Fisio-Motora na Universidade Federal de Santa Maria (UFSM), Santa Maria, RS, Brasil.

**Fisioterapeuta pela Universidade Federal de Santa Maria (UFSM), Santa Maria, RS, Brasil.

${ }^{* * *}$ Acadêmica de Fisioterapia na Universidade Federal de Santa Maria (UFSM), Santa Maria, RS, Brasil.

****Doutora em Ciências da Saúde pela Universidade de Brasília (UNB), Brasília, DF, Brasil. Professora titular no curso de Fisioterapia na Universidade Federal de Santa Maria (UFSM), Santa Maria, RS, Brasil.
} 


\section{Introdução}

O atual modelo de formação acadêmica dos profissionais da saúde ainda predomina a atenção curativa, centrado nas práticas hospitalares. No curso de fisioterapia, o seu histórico construído a partir da reabilitação ainda tem marcante influência na atuação dessa profissão, voltando o seu olhar para a atenção secundária e terciária á saúde ${ }^{2}$. Apesar disso, impulsionada pelas diretrizes curriculares nacionais dos cursos de graduação em fisioterapia a partir de 2002, diversos órgãos representativos profissionais têm alavancado novos conhecimentos e divulgação de trabalhos, possibilitando, nesse momento, um aprofundamento e reflexão sobre sua prática profissional na atenção primária através de ações que favoreçam a formação de um profissional capaz de atuar na saúde nos níveis de promoção, prevenção, preservação e recuperação da saúde do ser humano e prevenção de doenças e incapacidades $2,7,8$.

O contexto de ensino centrado no modelo curativo-reabilitador, com prioridade no ambiente ambulatorial e hospitalar não proporciona aos acadêmicos o conhecimento da realidade de vida da população e do seu adoecimento, levando a um consequente despreparo para atuar na atenção primária a saúde. Dessa forma destaca-se a importância da participação dos acadêmicos de fisioterapia em projetos, disciplinas, ou estágios que propiciem experiência nesse universo de atuação.

A disciplina complementar a graduação "Fisioterapia na Atenção Primária à Saúde", utilizando-se do projeto "Espaço de Vivências da Fisioterapia na Comunidade", proporcionou essa vivência aos alunos de fisioterapia da Universidade Federal de Santa Maria (UFSM). Na qual eles conheceram o contexto em que vivem os pacientes, os cuidadores e a família: como eles se relacionam entre si, as condições de vida e os recursos de que dispõem, e como as doenças modificam a sua rotina/seu modo de viver.

O projeto citado anteriormente tem como objetivos proporcionar espaço de vivências acadêmicas em práticas de fisioterapia na atenção primária de saúde; proporcionar aos acadêmicos do curso de Fisioterapia conhecer o universo de atuação da fisioterapia na comunidade; estimular a troca de experiências entre os acadêmicos e os profissionais do serviço de saúde; incentivar a produção de trabalhos científicos a partir das vivências e divulgar e oportunizar à comunidade o trabalho da fisioterapia na atenção primária à saúde.

O estudo de Silva e Da Ros ${ }^{2}$ demonstra a necessidade de inserção dos acadêmicos de fisioterapia em atividades voltadas à atenção básica, aproximando-os da realidade, ainda que de forma gradual, no decorrer do curso. A falta da inserção se reflete no perfil acadêmico do fisioterapeuta, onde muitos estão voltados apenas para o processo de reabilitação. 0 presente trabalho é justificado pela necessidade da educação dos profissionais de saúde ser pautada nos conhecimentos vividos, experimentados, pois esses permitem formar profissionais com capacidade de solucionar problemas, de contribuir para melhorar a situação de saúde da população. Impulsionando mudanças necessárias à construção de uma atuação profissional em Fisioterapia mais alargada, reforçando a necessidade de uma mudança de paradigma na formação acadêmica, que permita uma visão mais integral do ser humano e uma postura profissional mais humanizada e mais voltada para a realidade social

Saúde (Santa Maria), v.38, n.1, p. 121-128, Percepção dos acadêmicos sobre a vivência de fisioterapia na atenção primária a saúde onde os sujeitos estão inseridos. Além disso, o trabalho reflete um dos princípios das diretrizes curriculares para os cursos universitários da área da saúde onde consta que se deve "incentivar uma sólida formação geral, necessária para que o futuro graduado possa vir a superar os desafios de renovadas condições de exercício profissional e de produção do conhecimento"s. 


\section{Objetivo}

Relatar a percepção dos acadêmicos de fisioterapia sobre a vivência na disciplina de Fisioterapia na Atenção Primária.

\section{Metodologia}

O presente estudo consiste num pesquisa descritiva que aborda sobre a percepção dos acadêmicos sobre a vivência na disciplina complementar de graduação (DCG), denominada Fisioterapia na Atenção Primária a Saúde, ministrada aos acadêmicos do curso de Fisioterapia da Universidade Federal de Santa Maria (UFSM), realizada junto à comunidade abrangida pela Estratégia Saúde da Família - S. José, em Santa Maria/RS.

$\mathrm{Na}$ disciplina foram desenvolvidas 15 horas de atividades teóricas, com abordagens sobre princípios e organização da atenção básica no sistema único de saúde, publicações sobre fisioterapia na atenção primária à saúde, e 15 horas de práticas, ofertada no período de 03 a 18 de março de 2011. As atividades práticas consistiram em conhecer a rotina da unidade da ESF, visitas domiciliares e observação dos relatos dos pacientes e cuidadores que fazem uso dos atendimentos dos estagiários de fisioterapia. Nesta etapa contou-se com o acompanhamento multidisciplinar de agentes de saúde, de dois terapeutas ocupacionais e outros profissionais da equipe de saúde da unidade.

Participaram 14 acadêmicos, três deles matriculados no $9^{\circ}$ (último) semestre do curso e os demais no $7^{\circ}$ semestre, aqui identificados como sujeitos Ac. $19^{\circ}$ ou Ac. $17^{\circ}$. Descreve-se como resultados o conteúdo do parecer dos acadêmicos elaborado no final da disciplina, no qual relatam sobre a experiência, as facilidades, as dificuldades e sugestões e autoavaliação. Posteriormente procedeu-se a leitura qualitativa dos dados, tendo como referencial de análise a sistematização proposta por Minayo1.

\section{Resultados}

Os resultados foram sistematizados em três blocos, segundo os tópicos contidos no parecer, sendo estes: facilidades para aprendizagem, dificuldades e sugestões para as dificuldades, e autoavaliação sobre a experiência.

\section{Facilidades para aprendizagem}

Dentre as facilidades para aprendizagem, os acadêmicos consideraram que 0 conhecimento da unidade Estratégia de Saúde da Família (ESF) e da equipe multidisciplinar, bem como o conhecimento do ambiente domiciliar dos pacientes, permitindo uma visão prévia da realidade socioeconômica dos usuários, o que facilitará a atuação futura destes estudantes durante o período de estágio, além de uma possível oportunidade de trabalhar com atenção primária à saúde quando fisioterapeutas, como pode ser observado nos dizeres a seguir:

Saúde (Santa Maria), v.38, n.1, p. 121-128, 2012. Silva, M. M., et al. 
"Visitas simples e contato direto com pacientes em seus locais de moradia, ampliando e facilitando o conceito de globalidade que devemos considerar durante o tratamento terapêutico" (Ac. $\left.1 / 7^{\circ}\right)$.

"Conhecer as limitações do ambiente, aprendendo a adaptar as atividades à escassez de materiais e recursos" (Ac.5/70).

"Conhecer a realidade sócio-econômica do usuário e o contexto psicoemocional do mesmo e de seus cuidadores" (Ac.10/70).

Os acadêmicos indicaram como fatores positivos a valorização por parte dos usuários com o serviço de fisioterapia, o funcionamento da equipe, dentro e fora da ESF, o que faz com que haja o reconhecimento da necessidade de atuação dos profissionais de fisioterapia na rede de atenção básica. Apesar de a atuação do fisioterapeuta ser entendida como assistência no nível de atenção terciária; quando inserido na atenção primária, pode ser de grande valia para ações de promoção da saúde, prevenção de doenças e educação em saúde ${ }^{4}$.

Ainda, afirmam como facilidades para aprendizagem, a visão multiprofissional através da convivência com outros profissionais da residência multiprofissional (terapeuta ocupacional e enfermeiro), bem como dos Agentes Comunitárias de Saúde (ACS), e o contato prévio dos alunos com a comunidade em geral, antes do período de estágio supervisionado II, conforme as expressões a seguir:

"Observação de fatores sociais referentes ao ambiente familiar e da
comunidade; limitações físicas dos pacientes e do ambiente onde estão
inseridos" (Ac. $9 / 7^{\circ}$ ).
"A vivência no ambiente familiar, observando todas as particularidades de
cada indivíduo e sua família, permite um atendimento mais individualizado
durante o estágio e de acordo com a realidade de cada um, além da
oportunidade de um planejamento prévio das condutas a serem realizadas
posteriormente" (Ac. $\left.14 / 9^{\circ}\right)$.

Outra contribuição importante relatada pelos acadêmicos foram as aulas teóricas que permitiram a discussão sobre o trabalho que está sendo realizado na área de abrangência ESF - São José, o estudo sobre a atuação da fisioterapia na atenção primária a saúde. Também foi enriquecedor o acompanhamento por parte de profissionais da terapia ocupacional e a presença dos Agentes Comunitárias de Saúde durante as visitas domiciliares, que ampliaram a visão dos acadêmicos quanto às necessidades dos usuários.

\section{Dificuldades}

Os acadêmicos relataram dificuldades durante este período de atuação no ESF - São

Saúde (Santa Maria), v.38, n.1, p. 121-128, 2012. Percepção dos acadêmicos sobre a vivência de fisioterapia na atenção primária a saúde José, como a acessibilidade para chegar em alguns domicílios por serem de espaço reduzido ao número de estudantes, em certos momentos a dificuldade em contar com profissionais da Residência Multiprofissional para acompanhamento nas visitas domiciliares. 
O escasso contato com a equipe da ESF, oportunizando um reduzido número de discussões multiprofissionais, com exceção das ACS e a precariedade na infra-estrutura e recursos encontrados na unidade foi outra dificuldade observada pelos estudantes, apresentado na expressão a seguir. Segundo Barros5, o fisioterapeuta pode desenvolver atividades efetivas em todos os níveis de atenção à saúde dentro da equipe interdisciplinar. Porém, devido a aspectos de ordem político-econômicos e organizacionais, sua função é pouco divulgada e subutilizada, contudo, paulatinamente experiências isoladas em algumas regiões brasileiras mostram que a inserção da fisioterapia na ESF enriquece e desenvolve ainda mais os cuidados de saúde da população.

"Obstáculos para o desenvolvimento das atividades, como falta de instrumentos e equipamentos fisioterápicos, necessitando certo improviso e criatividade por parte dos acadêmicos" (Ac.10/7ㅇ).

Outra dificuldade refere-se a pausa do atendimento da fisioterapia nas férias letivas faz com que os usuários que necessitam deste acompanhamento regular tenham alguns prejuízos, também o fato das equipes de saúde da ESF estarem incompletas naquele período tornava precário o acompanhamento dos usuários.

As sugestões para solucionar as dificuldades encontradas pelos acadêmicos foram: a oferta da disciplina em outras situações, como por exemplo, com maior carga horárias ao longo do semestre letivo, permitindo, desse modo, que se realizem atendimentos, além das visitas domiciliares; que as visitas sejam realizadas no turno da manhã, onde o clima é mais favorável nesta época do ano, para um melhor rendimento por parte dos alunos e dos usuários; a implantação de atividades que possam ser realizadas pelos pacientes durante as férias pode contribuir para a manutenção do tratamento realizado durante o período letivo.

Além disso, sugeriram a presença de um membro de cada núcleo da residência multiprofissional acompanhando as visitas para permitir uma visão mais multidisciplinar sobre cada paciente, contribuindo para 0 atendimento e 0 enriquecimento do conteúdo abordado nas aulas práticas; a divisão da turma em grupos menores de acadêmicos em cada domicílio torna mais fácil e objetivo o contato dos acadêmicos com os usuários, e pode auxiliar na sugestão de adaptações domiciliares que estejam ao alcance da situação sócio econômica do usuário; e a realização da teoria em dias subsequentes da prática possibilitaria mais discussões em sala de aula voltadas para a realidade de cada usuário.

\section{Auto-avaliação sobre a experiência de aprendizagem na atividade}

Os acadêmicos relataram inúmeros ensinamentos adquiridos com a disciplina complementar a graduação Fisioterapia na Atenção Primária à Saúde, que contribuiu para 0 seu desenvolvimento no curso e seu crescimento como seres humanos. Segundo Moraes ${ }^{6}$, o atendimento domiciliar possibilita ao fisioterapeuta conhecer a realidade social, econômica, cultural e familiar do paciente podendo adequar e conduta e realizar as orientações necessárias, incluindo orientação e capacitação dos membros da família quanto à conduta a ser seguida com o mesmo. Os cuidados domiciliares repassados à família vão desde orientações de saúde em geral até técnicas de estímulo sensório-motor, termoterapia e cinesioterapia. 
Outro aspecto positivo foi o fato de conhecerem previamente alguns dos pacientes com quem irão atender no estágio curricular, no $9^{\circ}$ semestre no Curso. Assim poderem começar a pensar antecipadamente no plano de tratamento para estes pacientes, voltado para a realidade e condições de moradia que observaram durante as visitas. Também, consideram de grande importância conhecer um pouco da maneira como cada cuidador lida com o paciente para poder prestar orientações que possam contribuir nessa relação. Os futuros profissionais demonstraram grande interesse pela área estudada e puderam perceber 0 quanto é gratificante o carinho das pessoas visitadas e o quanto elas valorizam a sua futura profissão, conforme explicitado:

\begin{abstract}
"Uma experiência sem dúvida positiva, pois permitiu o conhecimento de uma área de atuação da fisioterapia pouco trabalhada no decorrer do curso. Observamos a realidade, as condições precárias em que vivem grande parte destes usuários que atenderemos futuramente. Estas visitas possibilitaram um contato prévio com estes usuários, o que ajuda-nos a pensar em um plano de tratamento e elaborar diferentes maneiras de trabalhar com estes pacientes dentro dos recursos disponíveis (...) "Desde já, ficou claro que precisaremos de criatividade e dedicação para realizar um bom trabalho e possibilitarmos melhores condições de vida a estes usuários. Por trás desta realidade, existe todo um contexto histórico cultural que difere um pouco da realidade a qual estamos acostumados. Além do conhecimento, esta disciplina nos trouxe uma lição de vida, porque com certeza aprendemos muito com estas pessoas, e ainda temos muito que aprender e ensinar com essa troca de experiência" (Ac.7/90).
\end{abstract}

"O desenvolvimento dessa disciplina foi de grande valia pois proporcionou aos acadêmicos a visão de um atendimento mais humanizado e integral, evidenciando a importância as visita domiciliar e do atendimento de fisioterapia que, através dos procedimentos da atenção dispendida e do olhar humanizado, proporcionam muito mais que a reabilitação cinéticofuncional. (...) O conhecimento da realidade local pôde nos trazer uma reflexão sobre como e o que desenvolveremos como atividade de tratamento fisioterápico, as dificuldades que encontraremos e de que forma podemos superá-las.(...) O carinho e o respeito demonstrado pelos usuários nos fazem perceber o quão importante somos no fazer saúde." (Ac. $4 / 7^{\circ}$ ).

\title{
Conclusão
}

Nesta experiência observou-se que os alunos ficaram satisfeitos em participar da disciplina complementar a graduação "Fisioterapia na Atenção Primária à Saúde", e puderam com isso identificar a importância da inclusão do fisioterapeuta no âmbito da atenção primária a saúde. Também foi constatado que o fisioterapeuta pode e deve atuar em outros campos de trabalho, além de clínicas e hospitais. Esta proposta de inserir os acadêmicos no ESF

Saúde (Santa Maria), v.38, n.1, p. 121-128, 2012. Percepção dos acadêmicos sobre a vivência de fisioterapia na atenção primária a saúde ISSN 2236-5834 qualifica não apenas o trabalho na saúde, como também melhora a qualidade de vida das pessoas envolvidas nesse processo. É válido salientar a ampliação das áreas de atuação da fisioterapia, que parte para uma visão mais global de saúde, dando enfoque não só para a reabilitação, mas atuando na promoção e na educação em saúde, o que pode representar redução de gastos do sistema de saúde e proporciona melhoria na qualidade de vida da população. 


\section{Referências bibliográficas}

1. Minayo MCS. O desafio do conhecimento: pesquisa qualitativa em saúde. $8^{\circ}$ ed. São Paulo: Hucitec, 2004.

2. Silva DJ, Da Ros MA. Inserção de profissionais de fisioterapia na equipe de saúde da família e Sistema Único de Saúde: desafios na formação. Ciênc saúde cole. 2007 Dez; 12 (6): 1673-1681.

3. Almeida M, Maranhão E. Diretrizes Curriculares Nacionais para os Cursos Universitários da Área da Saúde; Rede Unida, 2003

4. Besen CB, Netto MS, Da Ros MA, Silva FW, et al. A Estratégia Saúde da Família como objeto de Educação em Saúde. Saúde Soc. 2007 Jan; 16 (1): 57-68.

5. Barros FBM. O fisioterapeuta na saúde da população: atuação transformadora. Fisiobrasil; 2002.

6. Moraes JR, Campregher A, Stapait A, Bruse CF, Grando K, Santos LF. A Atuação da Fisioterapia no Programa de Saúde da Família. Reabilitar. 2001; 10: 21-6.

7. Portes LH, Caldas MAJ, et al. Atuação do fisioterapeuta na Atenção Básica à Saúde: uma revisão da literatura brasileira. Rev. APS. 2011 Mar; 14 (1): 111-9.

8. Da Costa JL, Pinho MA, et al. A fisioterapia no programa de saúde da família: percepções dos usuários. Revista Ciência \& Saúde. 2009 Jun; 2 (1): 2-7.

\section{Maíra Machado da Silva}

Endereço para correspondência — Alameda Jandyr Garcia, 678. Bairro Cassino. Rio Grande, RS.

E-mail: maira_dasi@hotmail.com

Currículo Lattes: http://buscatextual.cnpq.br/buscatextual/visualizacv.do?id=K4413600D6

Recebido em 07 de setembro de 2011.

Aprovado em 14 de junho de 2012. 


\section{Saúde (Santa Maria), v.38, n.1, p. 121-128,}

$$
2012 .
$$

Percep̧̧ão dos acadêmicos sobre a vivência de fisioterapia na atençăo primária a saúde

128 | ISSN 2236-5834 